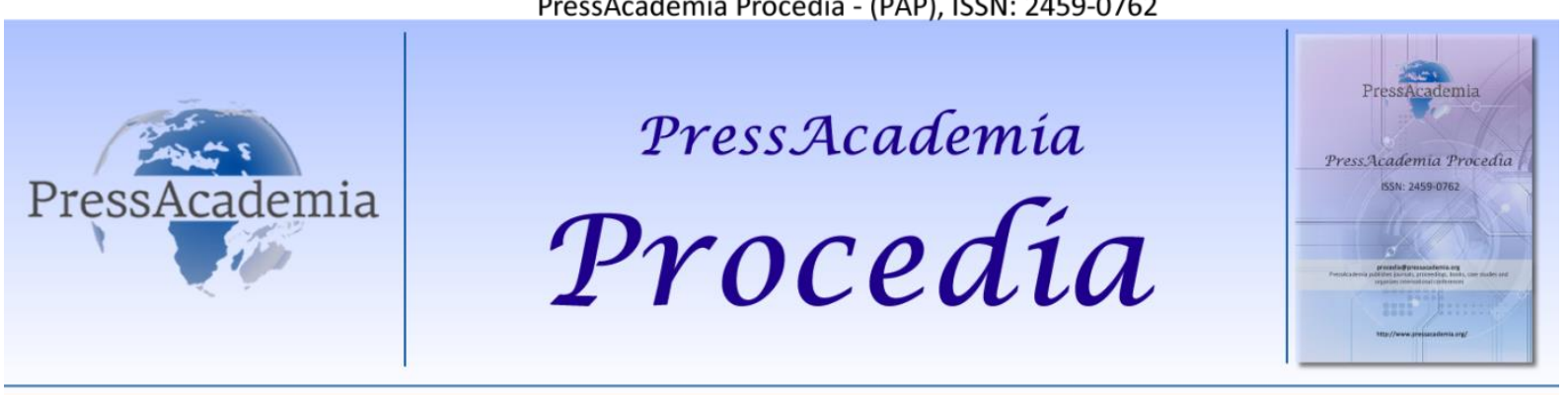

2nd World Conference on Technology, Innovation and Entrepreneurship

May 12-14, 2017, Istanbul, Turkey. Edited by Sefer Şener

\title{
PERFORMANCE ANALYSIS OF A COOPERATIVE COMMUNICATION SYSTEM USING MASSIVE MIMO
}

\author{
DOI: 10.17261/Pressacademia.2017.563 \\ PAP-WCTIE-V.5-2017(1)-p.1-9
}

Sidi Ethmane Amar ${ }^{1}$, Tansal Gucluoglu ${ }^{2}$

${ }^{1}$ Yildiz Technical University. sidijedou@gmail.com

${ }^{2}$ Yildiz Technical University. tansal@yildiz.edu.tr

\begin{abstract}
Massive MIMO will be an enabling technology for the deployment of the 5th generation of wireless systems. In the past three years, this technology has been put under the spotlight. Although there is a lot of research work on this topic, a number of challenges still need to be surpassed before reducing Massive MIMO to practice. In this paper, I studied and explored a cooperative communication scenario using massive MIMO at the base station. Based on the work previously done by the project WINNER and the Vienna LTE Simulator, we generated a propagation channel model and simulated the throughput of the system model using different types of schedulers and transmission modes.
\end{abstract}

Keywords: Massive MIMO, cooperation, scheduler, CoMP.

\section{INTRODUCTION}

In the last decade, data traffic has increased in a very exponential way due to the easy access to the internet driven by a dramatic growth and evolution of both wireless data consuming devices (like smartphones, tablets, and laptops) and broadband networks' infrastructure. In other words, the demand for wireless data traffic will increase even more dramatically in the foreseeable future. Thus, new disruptive technologies are required to meet this huge demand. The key factor to take into consideration in wireless data traffic is the wireless throughput (bits/s) which is defined as: Throughput = Bandwidth $(\mathrm{Hz}) \times$ Spectral efficiency (bits $/ \mathrm{s} / \mathrm{Hz}$ ). Evidently, to enhance this throughput, some novel technologies which can increase the bandwidth or the spectral efficiency or both should be explored.

Transmission with multiple-input multiple-output (MIMO) antennas is a well-known diversity technique to enhance the reliability of the communication. The more antennas the base station BS is equipped with, the more degrees of freedom are offered and hence, more users can simultaneously communicate in the same time-frequency resource. As a result, a huge sum throughput can be obtained.

With large antenna arrays, conventional signal processing techniques (e.g. maximum likelihood detection) become prohibitively complex due to the high signal dimensions. The main question is whether we can obtain the huge multiplexing gain with low-complexity signal processing and low-cost hardware implementation. Research has shown that the use of an excessive number of BS antennas compared with the number of active users makes simple linear processing nearly optimal in [1].

Due to the complexity and deployment consideration in practical scenarios at individual BSs, each BS site cannot be deployed with a large number of antennas. That means with a limited number of antennas, the inter-cell and intra-cell interference still exist if simple non-cooperative linear precoding is used individually in each base station site [2]. Cooperative massive MIMO [CM-MIMO] where multiple BSs cooperate together and form a distributed antenna array to serve multiple users simultaneously is an attractive alternative. In CM-MIMO, user data as well as CSI (channel state information) is shared among BSs that will provide more degrees of freedom for communication. Also, precoding can take into account inter-cell interference and thus mitigate inter-cell interference, which is especially critical for cell edge users that typically suffer more inter-cell interference. 
Table 1: The Cisco VNI Forecast - Historical Internet Context

\begin{tabular}{|c|l|}
\hline Year & Global Internet Traffic \\
\hline 1992 & 100 GB per day \\
\hline 1997 & 100 GB per hour \\
\hline 2002 & 100 GBps \\
\hline 2007 & 2,000 GBps \\
\hline 2015 & 20,235 GBps \\
\hline 2020 & 61,386 GBps \\
\hline
\end{tabular}

Source: Cisco VNI, 2016

Furthermore, CM-MIMO, where multiple BSs coordinate through the backhaul network, the bandwidth of the backhaul link and delay may create additional impairments on the system performance [3], [4]. In this paper, system level simulation performance of cooperative massive MIMO scenario (specifically with coordinated multipoint CoMP technique) is compared to other non-cooperative massive MIMO scenarios under the uniform framework of the LTE-A system. Here, the analysis is based on comparing the results of different simulations using the same setup, varying only the scheduler type and the transmission mode to see how it will affect the throughput and the spectral efficiency of the UEs. This analysis provides insight on the potential system performance that can be achieved by using cooperative massive MIMO. The rest of the paper is organized as follows. In Section 2, the 3GPP 3D channel model used for simulation is described. Section 3 presents the system simulation setup and simulation results. Finally, our conclusion is presented in Section 4.

\section{The 3GPP 3D Channel Model}

The 3GPP 3D channel model, used in this paper, characterizes wireless communication channels of typical European cities. It is a 3D geometric stochastic model, describing the scattering environment between BS and UE in both azimuth and elevation dimensions. The scatterers are represented by statistical parameters without having a real physical location. In 3GPP TR 36.873 [5], three scenarios, Urban Macro cell (UMa), Urban Micro cell (UMi) and UMa-high rise (UMa-H) are specified. They represent typical urban macro-cell and micro-cell environments. Both UMa and UMa-H scenarios, consider a BS height of $25 \mathrm{~m}$, thus surpassing the surrounding buildings. UMa-H also specifies such environments with one high-rise building per Evolved Base Station (eNodeB) sector. UMi, considers a BS height of 10m, lying below the rooftop level. All three environments are assumed to be densely populated with buildings and take into account both indoor-and outdoor UEs.

The 3GPP 3D channel model specifies three propagation conditions, Line-Of- Sight (LOS), Non-Line of Sight (NLOS) and Outdoor-to-Indoor (O-to-I). For each of these conditions it defines different parameters for mean propagation path loss, macroscopic fading and microscopic fading. All three scenarios in [5], UMa, UMi and UMa-H, consider $80 \%$ of the UEs to be located indoors. The probability of being in LOS is determined separately for indoor and outdoor UEs and depends on the height of the UE as well as the break point distance. The break point distance characterizes the gap between transmitter and receiver at which the Fresnel zone is barely broken for the first time [6]. For an indoor UE, LOS refers to the signal propagation outside the building in which the UE is located.

For each UE location, large scale parameters are generated according to its geographic position as well as the propagation conditions at this location. The large-scale parameters incorporate shadow fading, the Ricean K-factor (only in the LOS case), delay spread, azimuth angle spread of departure- and arrival, as well as zenith angle spread of departure and arrival. The small-scale parameters incorporate delays, cluster powers as well as angles of departure and -arrival in azimuth and elevation direction, respectively. The model considers $\mathrm{N}$ clusters of scatterers, where each cluster is resolvable to $\mathrm{M}$ paths. A simplified sketch of the model is given in Fig. 1. 
Figure 1: Scattering concept in the 3D model. Figure demonstrates a link that is resolvable to $M$ paths. Elevation- and azimuth angles at BS and UE are denoted as $\theta$ and $\phi$, respectively [8]

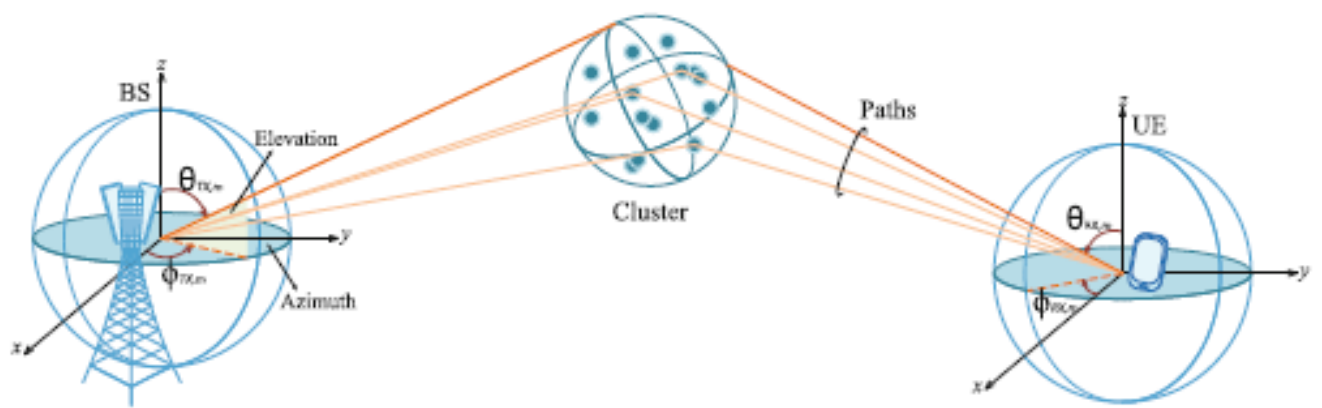

\section{SYSTEM LEVEL SIMULATION}

\subsection{Simulation Setup}

The macro-sites are arranged according to a hexagonal grid with an inter-site distance of $500 \mathrm{~m}$. Each site is equipped with three sector eNodeBs; each eNodeB employs $4 \times 2$ antenna configuration on the macro-site. The minimum distance between a UE and its associated eNodeB is $35 \mathrm{~m}$.

Figure 2: Hexagonal-grid tri-sector macro-cell scenario. [8]

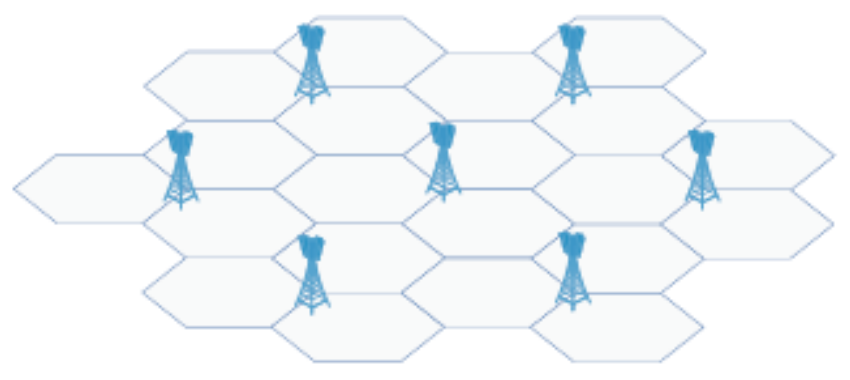

In the table 2. below, there is a detailed list of the employed simulation settings. We simulate at the center frequency of 2 $\mathrm{GHz}$ and an LTE bandwidth of $10 \mathrm{MHz}$; the total transmit power per eNodeB is $40 \mathrm{~W}$. The eNodeBs on the macro-site employ directional antennas. The signal propagation conditions follow the model of the 3GPP 3D channel, as mentioned previously. Each UE has two receive antennas and employs a Zero-forcing receiver ZF within each eNodeB sector. We assume 10 active users which are uniformly distributed and move at the speed of $5 \mathrm{Km}$ per hour. We employ closed loop spatial multiplexing CLSM corresponding to transmission mode 4 of LTE. The feedback comprises channel quality indicator CQI, precoding metrics indicator PMI, and rank indicator RI. The feedback is delayed by three transmission time intervals TTI and computed with perfect channel knowledge. Resources are assigned according to CoMP scheduler. We assume a simple full buffer traffic model and simulate over a total length of 20TTI.

Table 2: Simulation Parameters

\begin{tabular}{l|l}
\hline PARAMETER & VALUE \\
\hline CARRIER FREQUENCY & $2 \mathrm{GHz}$ \\
LTE BANDWIDTH & $10 \mathrm{MHz}$ \\
MACRO-SITE DEPLOYMENT & Hexagonal grid \\
SCENARIOS & $3 \mathrm{D}-\mathrm{UMa}$ \\
ENODEB ANTENNA HEIGHT (UMA) & $25 \mathrm{~m}$ \\
ENODEB TRANSMIT ANTENNA CONFIGURATION & $N_{\mathrm{Tx}}=4$, each has 8 ports
\end{tabular}




ENODEB RECEIVE ANTENNA CONFIGURATION
ENODEB ANTENNA ELEMENTS PER PORT
POLARIZED ANTENNA MODELING
ENODEB ANTENNA POLARIZATION
UE ANTENNA POLARIZATION
VERTICAL ANTENNA ELEMENT SPACING
HORIZONTAL ANTENNA ELEMENT SPACING
MAXIMUM ANTENNA ELEMENT GAIN
UE ANTENNA PATTERN
ELECTRICAL DOWNTILT
UE DISTRIBUTION
NUMBER OF ENODEBS
ENODEB TRANSMIT POWER
LTE TRANSMISSION MODE (MIMO MODE)
ANTENNAS PER UE
RECEIVER TYPE
ACTIVE UES PER ENODEB
UE SPEED
TRAFFIC MODEL
CHANNEL KNOWLEDGE
FEEDBACK
FEEDBACK DELAY
SCHEDULER
SIMULATION LENGTH

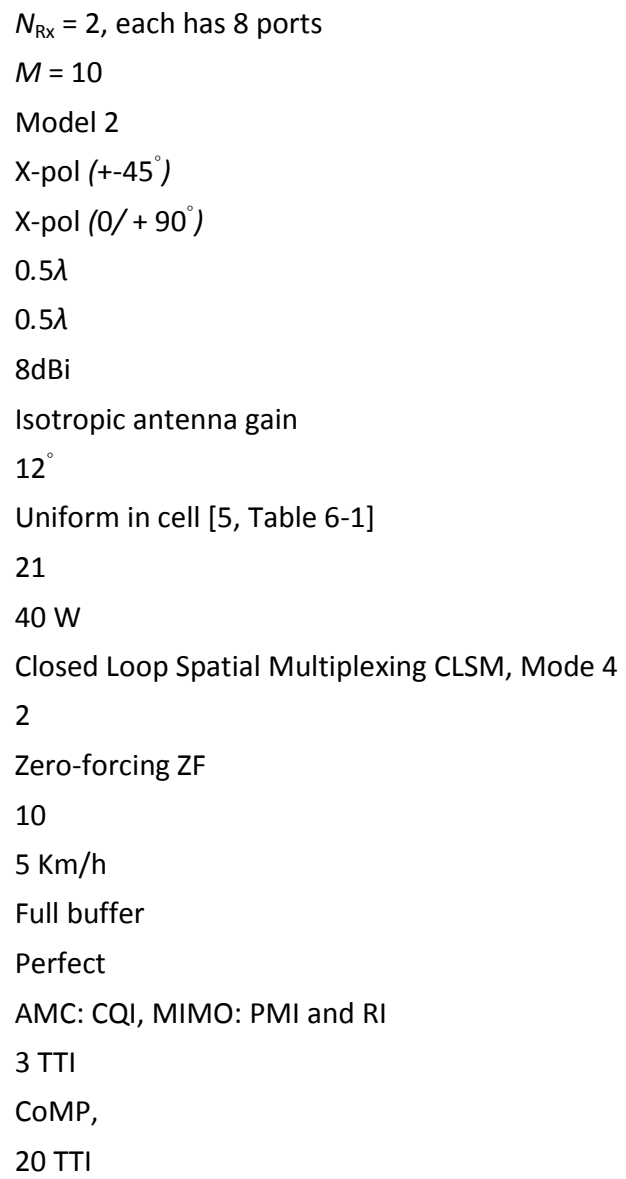

\subsection{Simulation Results}

The simulator initially generates macro-site and the associated eNodeBs, then it computes the site specific pathloss and shadow fading maps. Based on the pathloss and the shadow fading maps, the simulator computes a maximum SINR map as shown in the upper left corner of Fig. 3 below. This map is then applied to calculate the UEs associations regions as shown in the lower right corner of Fig. 3. The spectrum patterns stem from the shadow fading. In each association region, the simulator uniformly distributes the specified number of UEs, in our case 10; then it initializes the schedulers and the resource blocks for each eNodeB. The next step is to calculate the fast fading channels. 
Figure 3: Macroscopic and Shadow Fading
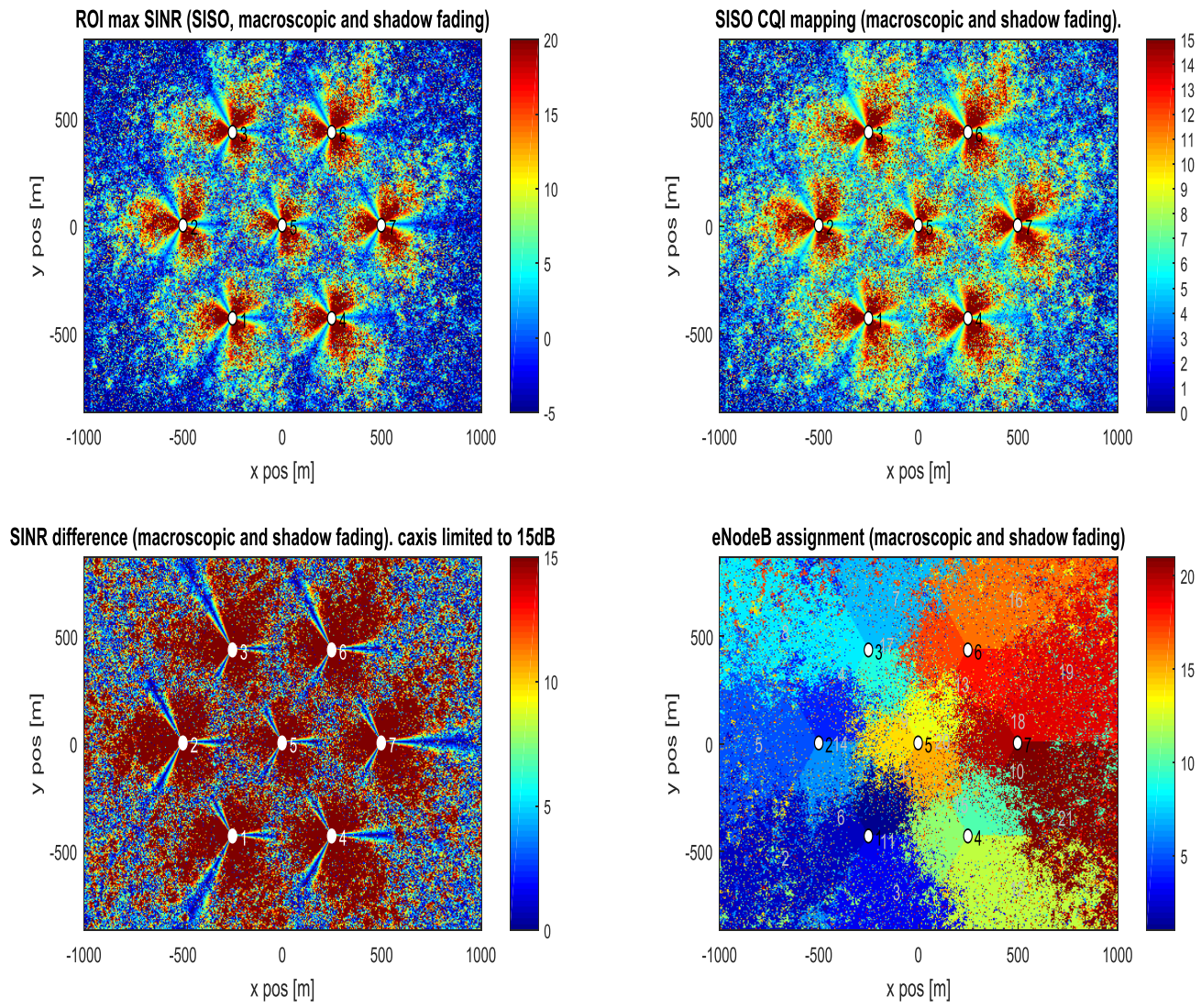

Our simulation employs mutual information based SNR mapping that maps the vector of post equalization SINR values from the link quality model to an effective SNR value in terms of mutual information; then using a lookup table and selecting the curve for the applied coding and modulation scheme allows to predict a block error ratio of the transport block.

Figure 4: SNR-to-CQI Mapping - CQI BLER Curves

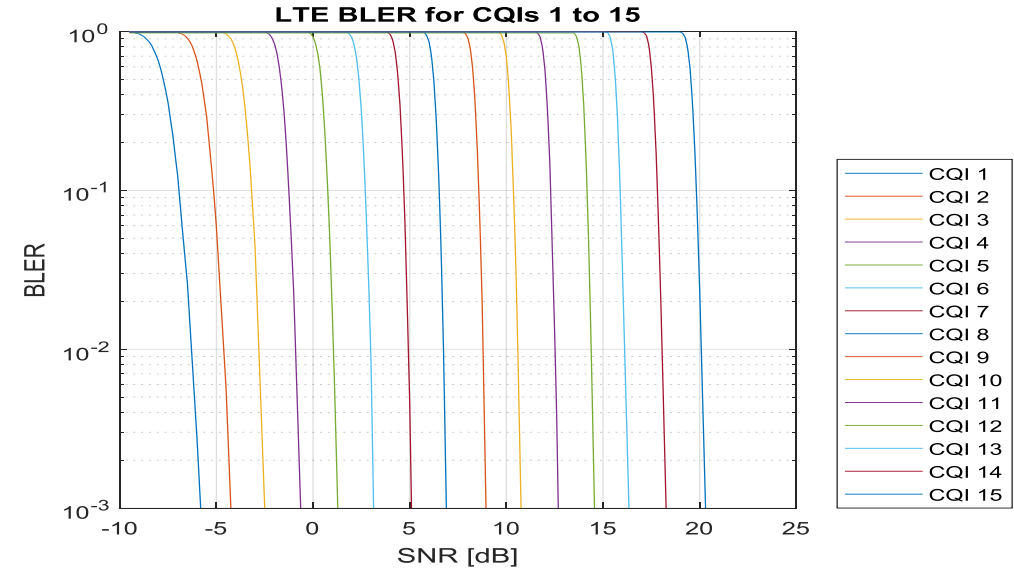


Figure 5: CQI Mapping Obtained from the $10 \%$ BLER Points
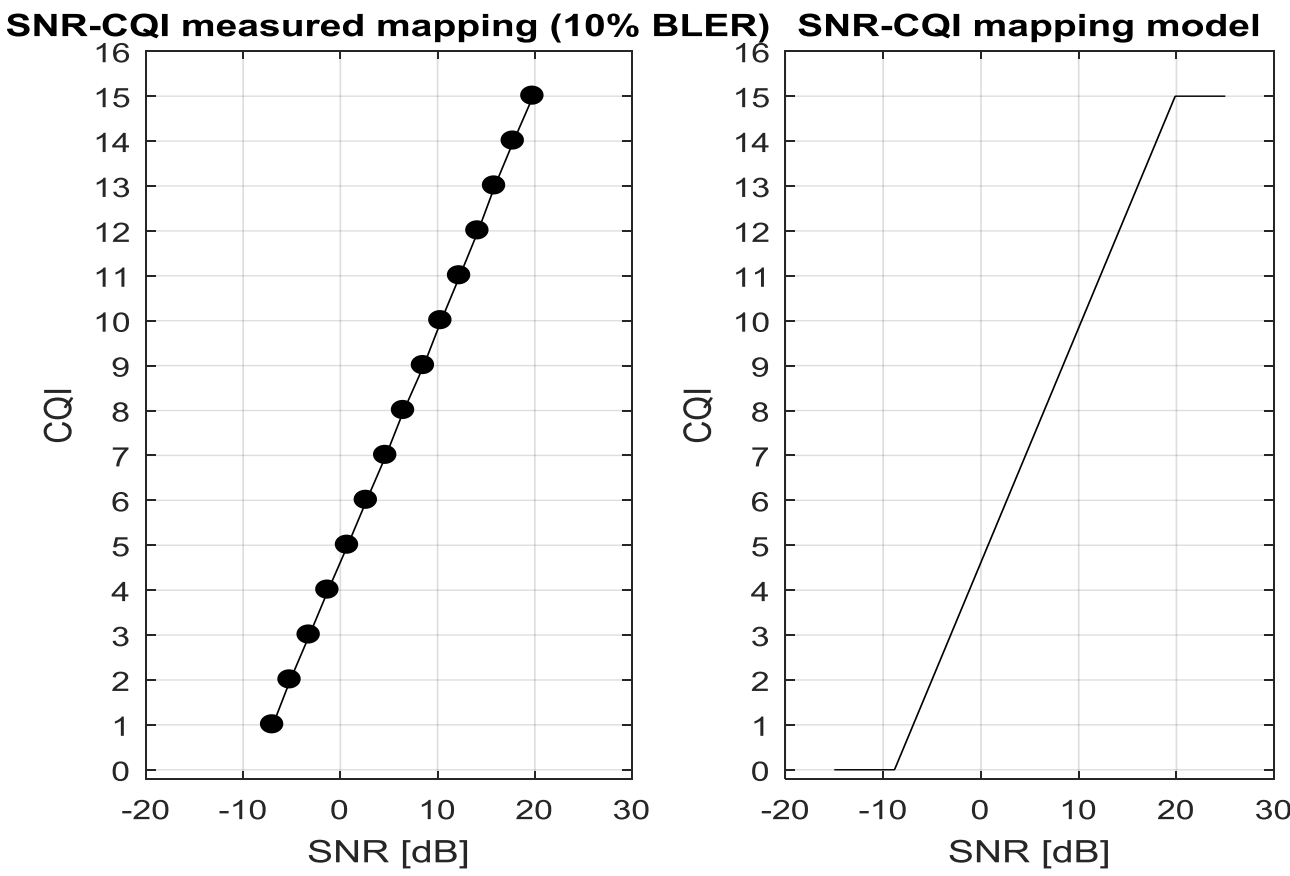

The presented curves were obtained with the Vienna LTE Advanced Link Level Simulator and form the only computationally costly physical layer evaluation. Eventually, combining the block error ratio with the size of transport block in bits yields the effective throughput.

\section{Figure 6: eNodeBs and UEs Positions}

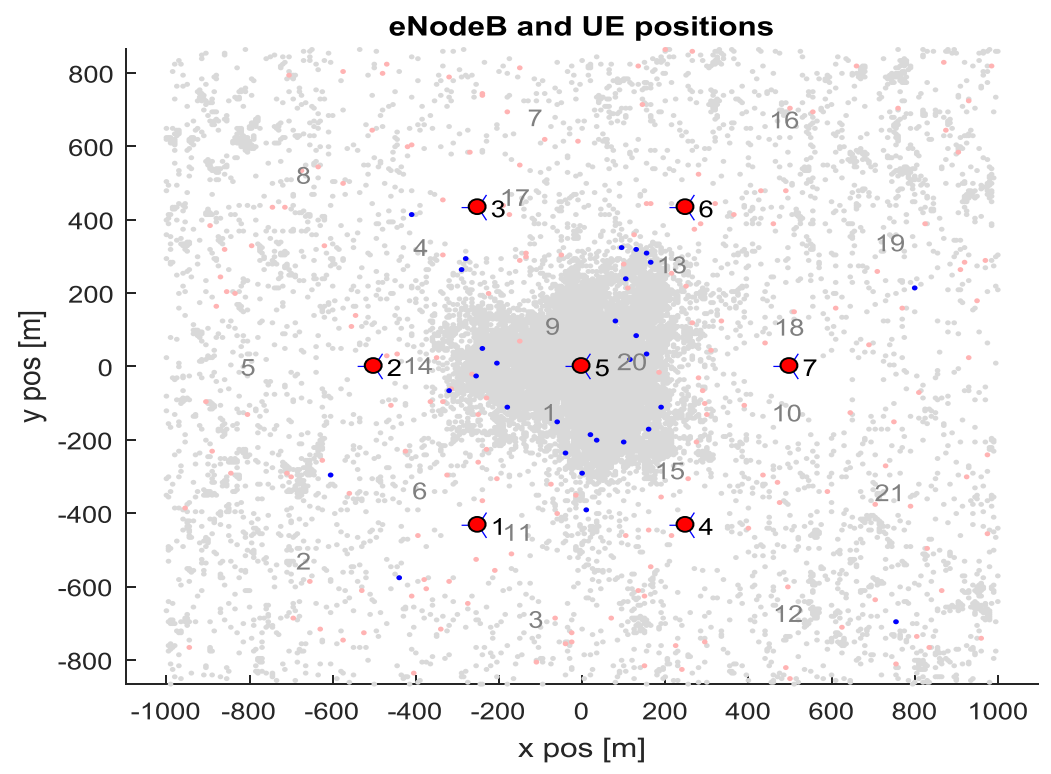

The figure Fig. 6 shows the simulated network with the bold red dots denoting the macro-site, and the blue and grey dots correspond to the UEs.

Figure 7 contains empirical cumulative distribution function for the average UE throughput, the average UE spectral efficiency and the UE wideband SINR. It also provides plots for the average UE throughput and the UE spectral efficiency Vs. the wideband SINR. 

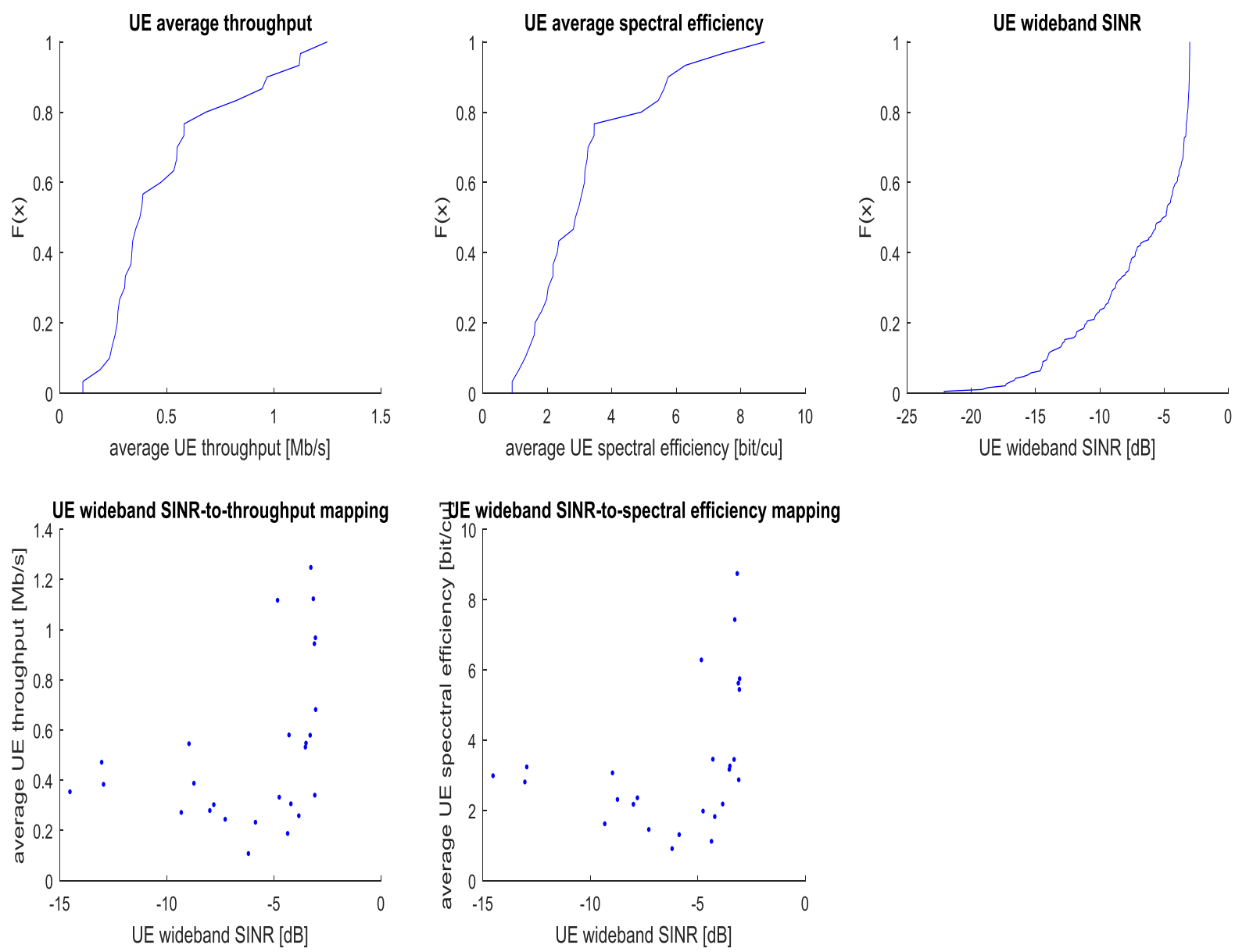

Figure 7: Plots show the throughput ECDF for the average UE throughput (upper-left), spectral efficiency (upper-middle), wideband SINR (upper-right); the mapping between the wideband SINR and the average throughput for each UE (lowerleft) and the mapping between the wideband SINR and the spectal efficiency (lower-right).

\subsection{Comparison and Evaluation of Results}

In this part, we evaluate the results of different simulations under the same previous configuration. Using the transmission mode closed loop spatial multiplexing CLSM with three types of schedulers round robin, proportional fair, and CoMP in contrast to the transmission mode MU-MIMO with round robin scheduler, we obtained the aggregate results in the table below. Table. 3 contains the UE throughput values, the 95\% (peak), average, and 5\% (edge); the average UE spectral efficiency and the average number of resource blocks per TTI and UE; as well as the statistics of mean RB occupancy and Fairness index.

Table 3: Results of Different Simulations

\begin{tabular}{|l|l|l|l|l|l|l|l|}
\hline $\begin{array}{l}\text { Transmission } \\
\text { Mode }\end{array}$ & Scheduler & $\begin{array}{l}\text { Peak/Avg./edge UE } \\
\text { throughput (Mb/s) }\end{array}$ & $\begin{array}{l}\text { Avg. UE } \\
\text { spectral eff. } \\
\text { (bit/cu) }\end{array}$ & $\begin{array}{l}\text { Avg. } \\
\text { RBs/TTI/UE } \\
\text { (RBs) }\end{array}$ & $\begin{array}{l}\text { Average cell } \\
\text { throughput } \\
\text { (Mb/s) }\end{array}$ & $\begin{array}{l}\text { Mean RB } \\
\text { occupancy }\end{array}$ & $\begin{array}{l}\text { Fairness } \\
\text { index }\end{array}$ \\
\hline CLSM & round robin & $3.51 / 1.49 / 0.15$ & 1.77 & 5 & 14.88 & $100 \%$ & 0.490905 \\
\hline CLSM & Prop Fair Sun & $3.81 / 1.21 / 0.12$ & 1.71 & 5 & 12.15 & $100 \%$ & 0.423385 \\
\hline CLSM & CoMP Intra-site & $2.07 / 0.67 / 0.11$ & 2.38 & 1.67 & 6.73 & $36.67 \%$ & 0.568229 \\
\hline CLSM & CoMP Global & $1.12 / 0.50 / 0.19$ & 3.28 & 0.92 & 5.04 & $25.61 \%$ & 0.737139 \\
\hline MU-MIMO & round robin MU & $3.79 / 1.40 / 0.14$ & 1.52 & 5.32 & 13.96 & $25.62 \%$ & 0.621665 \\
\hline
\end{tabular}


According to the comparative results shown in Table 3. Above, our cooperative scenario yields the least average UE and cell throughputs but it has slightly better edge UE throughput and in terms of resources allocation, it has the best average UE spectral efficiency and fairness index.

\section{Figure 8: UE Average Throughput}

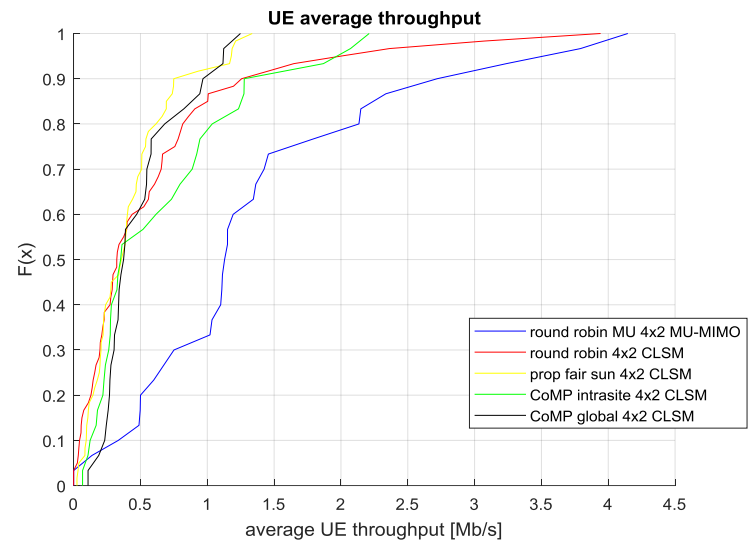

Figure 9: UE Average Spectral Efficiency

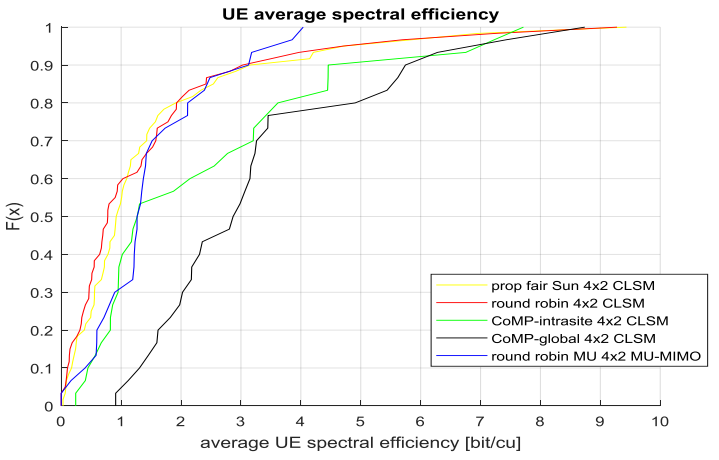

Figure 10: UE Wideband SINR

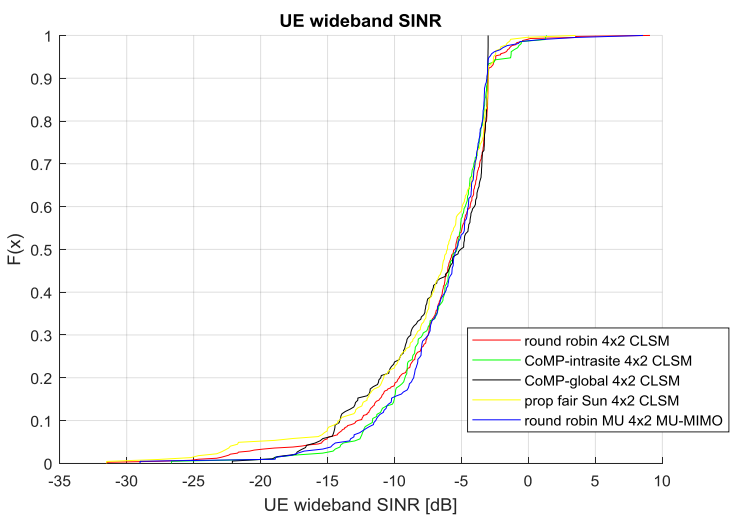

\section{CONCLUSION}

In this paper, downlink system level simulation performances of noncooperative and cooperative massive MIMO scenarios were evaluated and compared based upon current LTE-A system, considering different transmission schemes and scheduling types. The analysis of the results shows that the deployed cooperative scenario has better performance for the 
cell edge users and it significantly improves the spectral efficiency of the system, whereas the cell average throughput is degraded owing to the power imbalance of the cell center users. These results go in accordance with the main reason for which the DL/UL Coordinated Multi-Point transmission/reception (CoMP) technique was introduced; which is to improve the system capacity and cell edge user throughput.

\section{REFERENCES}

[1] F. Rusek, D. Persson, Buon Kiong Lau, E. G. Larsson, T. L. Marzetta, and F. Tufvesson, “Scaling Up MIMO: Opportunities and Challenges with Very Large Arrays," IEEE Signal Processing Magazine, vol. 30, no. 1, pp. 40-60, Jan. 2013.

[2] C. He and R. D. Gitlin, "Limiting Performance of Massive MIMO Downlink Cellular Systems," in Information Theory and Applications Workshop (ITA), Feb. 2016.

[3] R. W. Heath, T. Wu, Y. H. Kwon, and A. C. K. Soong, "Multiuser MIMO in Distributed Antenna Systems with Out-of-Cell Interference," IEEE Transactions on Signal Processing, vol. 59, no. 10, pp. 4885-4899, Oct. 2011.

[4] R. Zakhour and S. V. Hanly, "Base Station Cooperation on the Downlink: Large System Analysis," IEEE Transactions on Information Theory, vol. 58, no. 4, pp. 2079-2106, Apr. 2012.

[5] $3^{\text {rd }}$ Generation Partnership Project (3GPP). Study on 3D channel model for LTE, 3rdGeneration Partnership Project (3GPP), TR 36.873 (2014)

[6] H. Masui, T. Kobayashi, M. Akaike, Microwave path-loss modeling in urban line-of-sight environments. IEEE J. Sel. Areas Commun. 20(6), 1151-1155 (2002). doi :10.1109/JSAC. 2002.801215

[7] Chao He and Richard D. Gitlin "System Performance of Cooperative Massive MIMO Downlink 5G Cellular Systems" Department of Electrical Engineering University of South Florida Tampa, Florida 33620, USAIEEE WAMICON 2016 April 11-13, 2016

[8] Markus Rupp, Stefan Schwarz and Martin Taranetz "The Vienna LTE-Advanced Simulators Up and Downlink, Link and System Level Simulation" ISSN 1860-4862 ISSN 1860-4870 (electronic) Signals and Communication Technology ISBN 978-981-10-0616-6 ISBN 978981-10-0617-3 (eBook) DOI 10.1007/978-981-10-0617-3

[9] M. Taranetz, T. Blazek, T. Kropfreiter, M. Mller, S. Schwarz, M. Rupp: "Runtime Precoding: Enabling Multipoint Transmission in LTEAdvanced System-Level Simulations"; IEEE Access, 3 (2015), 725 - 736 